\title{
The Study of the Effects of Mobility Trends on the Statistical Models of the COVID-19 Virus Spreading
}

\author{
Davit Gondauri ${ }^{1 \star}$, Mikheil Batiashvili ${ }^{1}$
}

${ }^{1}$ Business and Technology University, GEORGIA

*Corresponding Author: dgondauri@gmail.com

Citation: Gondauri D, Batiashvili M. The Study of the Effects of Mobility Trends on the Statistical Models of the COVID-19 Virus Spreading. Electron J Gen Med. 2020;17(6):em243. https://doi.org/10.29333/ejgm/8212

\begin{tabular}{|c|c|}
\hline ARTICLE INFO & ABSTRACT \\
\hline Received: 22 Apr. 2020 & The history of the Pandemics makes a significant impact on the memory and behavior of the affected \\
\hline Accepted: 25 Apr. 2020 & $\begin{array}{l}\text { communities. It is important to study the connection between human mobility and the spread of viral infection. } \\
\text { Specifically, we aimed to investigate whether there was a correlation between Mobility Trends and the spread of } \\
\text { Covid-19 virus. Thus, in the conclusion it should be noted that the intensity of pedestrians, traffic and transit traffic } \\
\text { during the study period, on average, after } 15-20 \text { days, affected the spread of the virus. If there was a positive slope } \\
\text { and correlation coefficient between the variables presented in the period } 22.01 .2020-11.03 .2020 \text { (before the } \\
\text { announcement of the pandemic), in the period } 12.03 .2020 \text { - } 14.04 .2020 \text { (after the announcement of the pandemic) } \\
\text { the slope and correlation coefficients received negative values between the study variables, which indicates That } \\
\text { on average, after } 15-20 \text { days, Due to the intensity of the movement, the center of the virus spread is identified, and } \\
\text { the intensity of the movement itself is decreased. }\end{array}$ \\
\hline
\end{tabular}

Keywords: COVID-19, mobility trends, regression slope, pearson correlation

\section{INTRODUCTION}

The history of the Pandemics makes a significant impact on the memory and behavior of the affected communities. The outbreak of the COVID-19 virus in Wuhan, Hubei Province, China was followed by the rapid spreading from its origin (1). For the time being the causative virus has been named as a severe acute respiratory syndrome coronavirus 2 (SARS-CoV-2) and the relevant infected disease has been named as coronavirus disease 2019 (COVID-19) by the World Health Organization respectively. Conferring to the daily report of the World Health Organization, the epidemic of SARS-CoV-2 so far registered 118319 cases and 4292 deaths in 113 countries according to the reported numbers by March 11, 2020 and the World Health Organization declared the outbreak of a pandemic (https://www.who.int/docs/default-source/corona viruse/situation-reports/20200311-sitrep-51-covid-19.pdf?sfvr sn=1ba62e57_10) (2). Following the outbreak significant behavioral, clinical, and state interventions have been undertaken to ease the epidemic and prevent the transmittal of the virus in remaining human populations in China and worldwide. It remains unclear how these governmental interventions, including travel restrictions, affected COVID-19 spread in China. We use real-time mobility data from Wuhan and detailed case data including travel history to elucidate the role of case introduction on transmission in cities across China and determine the impact of the imposed control measures. The spatial distribution of COVID-19 cases in China was explained well by human mobility data. Following the execution of the government control measures, this correlation of the spreading dropped and growth rates became negative in most places, although shifts in the demographics of the reported cases were still indicative of local chains of transmission outside Wuhan. The following study shows that the radical and sever control procedures employed in China substantially mitigated the spread of COVID-19 virus (3). Limiting the social contacts of these individuals was crucial for COVID-19 control, because patients with no or mild symptoms can spread the virus (1).

As it is known from the previous examples, the importance of human mobility for malaria elimination was evident in earlier elimination attempts. However, the malaria re-emerged due to the failed surveillance systems that had to account the movements of the affected human populations (4).

Human mobility is a major factor in the spread of vectorborne diseases such as dengue even on the short scale corresponding to intra-city distances. In this study we finally discuss the advantages and the limits of mobile phone data and potential alternatives for assessing valuable mobility patterns for modeling vector-borne diseases outbreaks in the cities (5). However, it is important consumers perceive strongly the risks of a pandemic, they can influence the beliefs of those around them about the risks they are facing, and it also promotes their attitude to cope with threats from the external environment (6).

Thus, it is important to study the connection between human mobility and the spread of viral infection. Specifically, we aimed to investigate whether there was a correlation between Mobility Trends and the spread of COVID-19 virus. 


\section{MATERIALS AND METHODS}

For this purpose, we used the Mobility Trends from the reports of the company Apple. The given reports are published daily and reflect requests for directions in Apple Maps. Privacy is one of our basic values, so the maps doesn't associate the user's data with the individual Apple ID, and Apple doesn't keep a history of where the user has been traveling. In many countries/regions and cities, relative traffic volume has increased since January $13^{\text {th }}$, consistent with the normal, seasonal usage of Apple Maps application. Day of week effects are important to normalize as the individual uses this data. Data that is sent from users' devices to the maps service is associated with random, rotating identifiers so Apple doesn't have a profile of individuals movements and its search history. Apple Maps has no demographic information about the application users, so any statements about the representativeness of the usage against the overall population cannot be made.

Data on confirmed cases of the COVID-19 virus transmission have been obtained from the World Health Organization's official daily reports. The data covers the number of people infected with COVID-19 virus since January 22 to April 14, 2020. As of January 22, there were 580 cases of reported infections, and as of April 14, the number reached 1 844863 , Including 71779 Total confirmed cases.

We calculated the results, and analyzed the data using regression slope and Pearson correlation. The regression slope method implies how much of a second variable changes by $1 \%$ of one variable. We have observed the global impact of pedestrian, machine and transit traffic on the impact of the spread of the virus, in particular the 1\% change in the cumulative number of confirmed infected cases. Correlation coefficients are scaled such that they range from -1 to +1 , where 0 indicates that there is no linear or monotonic association, and the relationship gets stronger and ultimately approaches a straight line (Pearson correlation) (7).

Pearson's product moment correlation coefficient is denoted as $\mathrm{Q}$ for a population parameter and as $r$ for a sample statistic. It is used when both variables being studied are normally distributed. This coefficient is affected by extreme values, which may exaggerate or dampen the strength of relationship, and is therefore inappropriate when either or both variables are not normally distributed. For a correlation between variables $x$ and $y$, the formula for calculating the sample Pearson's correlation coefficient is given by

$$
r=\frac{\sum_{i=1}^{n}\left(x_{i}-x\right)\left(y_{i}-y\right)}{\sqrt{\left[\sum_{i=1}^{n}\left(x_{i}-\bar{x}\right)^{2}\right]\left[\sum_{i=1}^{n}\left(y_{i}-\bar{y}\right)^{2}\right]}}
$$

where $x i$ and $y i$ are the values of $x$ and $y$ for the ith individual (8). We even used the Pearson correlation to determine the correlation between Mobility Trends and the total amount of infection.

For the study purposed, we have also selected the 8 countries which as of date April 15, 2020, have the highest numbers of the confirmed cases of the Corona Virus. Those countries are: USA (Total confirmed cases - 578268), Spain (Total confirmed cases - 172541), Italy (Total confirmed cases
- 162488), Germany (Total confirmed cases - 12758), France (Total confirmed cases - 102533), The United Kingdom (Total confirmed cases - 93877), Turkey (Total confirmed cases 65111) and Belgium (Total confirmed cases - 31119). At the same time, the Mobility Trends of these countries were recorded by the Apple. We also selected one additional country - New Zealand, which was characterized by a reliable decrease in the spread of the virus (Total cases - 1386; Daily cases - 20; Total recovered cases - 728). The data covers the number of Mobility Trends from January 13 to April 14, 2020. The data were divided into two periods before the pandemic (before March 11, 2020) and after the pandemic. We calculated the slope, correlation, and determination coefficients between intensity of walking, driving, transit and the prevalence of the virus according to the data of the given countries from January 22 to March 11 and from March 11 to April 14.

Limitation of the study provided we considered only a linear relationship between the variables. Naturally there are other factors that influence the number of infected cases that we disregarded in the study.

\section{RESULTS AND DISCUSSION}

The study is based on the COVID-19 statistical study based on Apple Maps data, which shows the movement of citizens by walking, by driving and transit as globally as in the following countries: USA, Italy, Spain, France, Germany, The United Kingdom, Belgium, Turkey, New Zealand.

- Walking - the volume of the spread of the virus. According to the statistical model, between January 22 and March 11, before the announcement of the pandemic, the slope between the number of pedestrians and the spread of the virus was $2.56 \%$, which means that when the spread of the virus increased by $1 \%$, the intensity of pedestrians increased. By $2.56 \%$. As for March 12-April 14, Slope is already negative among the already mentioned variables 2.61 times. The results already indicate that in the second phase of the virus spread (March 12 - April 14) in parallel with the $1 \%$ increase in the spread of the virus, the intensity of pedestrians decreased by 2.61 times. As for the correlation between the presented variables, it was $-75 \%$ for the mentioned period, and its determination coefficient $\left(R^{\wedge}\right.$ 2 ) is 56.28 .

- Driving- the volume of the spread of the virus. From January 22 until the announcement of the pandemic (March 11), the slope between the intensity of traffic and the extent of the spread of the virus was $-1,156$ times. This indicates that during this period, when the spread of the virus increased by $1 \%$, the intensity of traffic increased by 1,156 times. As for March 12-April 14, Slope is already negative among the already mentioned variables -2.61 times. In the second phase of the spread of the virus (March 12 - April 14) in parallel with the $1 \%$ increase in the spread of the virus, the intensity of traffic decreased by 3.18 times. As for the correlation between the presented variables for the mentioned period, it was $-77.9 \%$, and its determination coefficient $\left(R^{\wedge} 2\right)$ is $60.69 \%$.

- Transit - the volume of the spread of the virus. During the study it was identified that between the January 22 and March 11 (March 11), the intensity of transit traffic and the prevalence of the virus, the number of slips was 2.76 . This data indicates that during this period, when the prevalence of the virus increased by $1 \%$, the intensity of transit traffic 


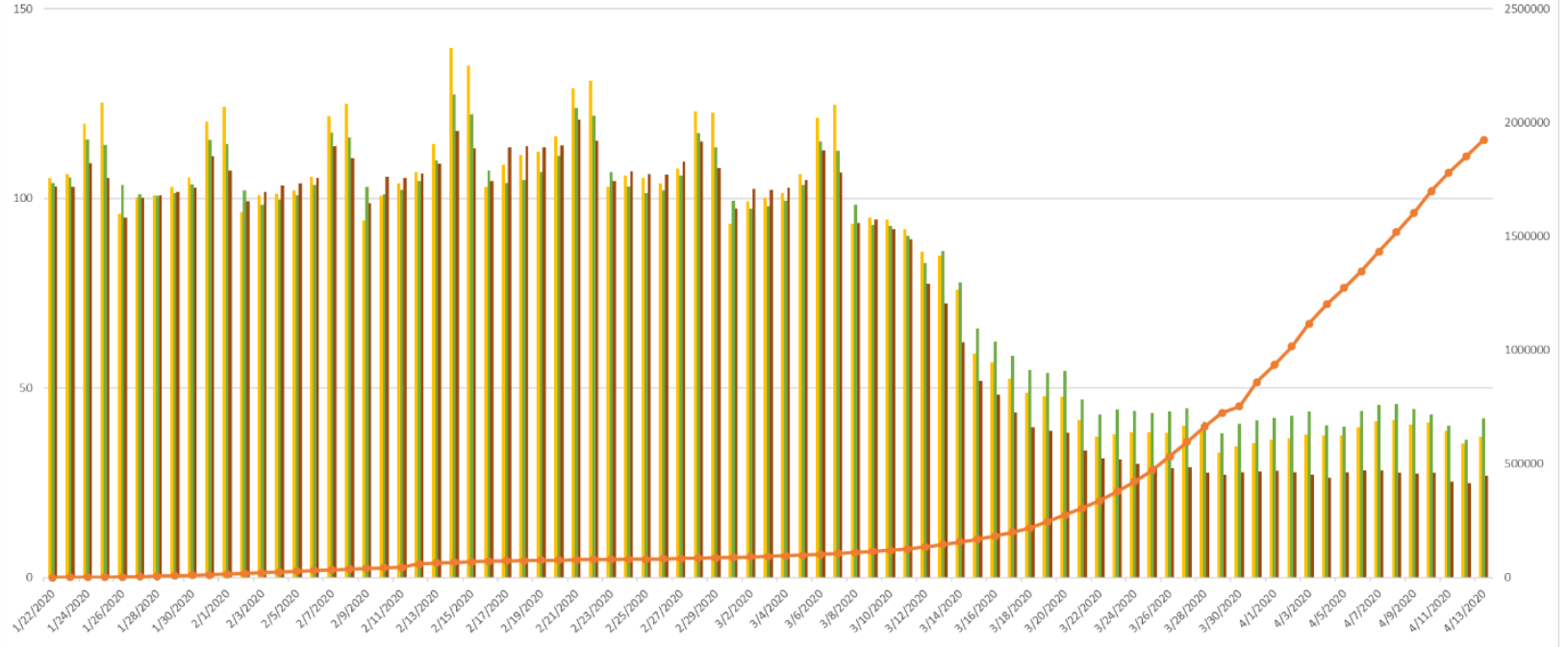

Figure 1. The cumulative number of globally confirmed cases of globally infected Covid-19 virus is given by day from January 22 to April 14; Graphically, the change in the number of pedestrians (Yellow columns), cars (Green columns), transit traffic (Red columns)

Table 1. Slope, correlation and determination coefficients obtained by Apple Maps traffic intensity by the country

\begin{tabular}{|c|c|c|c|c|c|c|c|c|c|c|}
\hline \multirow{2}{*}{ Country } & \multirow{2}{*}{ Date } & \multicolumn{3}{|c|}{ Slope Total Cases to } & \multicolumn{3}{|c|}{ Correl Total Cases to } & \multicolumn{3}{|c|}{$R^{\wedge} \mathbf{2}$} \\
\hline & & walking & driving & transit & walking & driving & transit & walking & driving & transit \\
\hline \multirow{2}{*}{ USA } & $22.01 .2020-11.03 .2020$ & 1.040 & 2.653 & -1.415 & \multirow{2}{*}{-0.608} & \multirow{2}{*}{-0.654} & \multirow{2}{*}{-0.682} & \multirow{2}{*}{0.370} & \multirow{2}{*}{0.427} & \multirow{2}{*}{0.465} \\
\hline & $12.03 .2020-14.04 .2020$ & -6.099 & -6.449 & -5.096 & & & & & & \\
\hline \multirow{2}{*}{ Spain } & $22.01 .2020-11.03 .2020$ & -2.097 & -5.102 & 2.985 & \multirow{2}{*}{-0.706} & \multirow{2}{*}{-0.737} & \multirow{2}{*}{-0.723} & \multirow{2}{*}{0.499} & \multirow{2}{*}{0.543} & \multirow{2}{*}{0.523} \\
\hline & $12.03 .2020-14.04 .2020$ & -1.564 & -2.140 & -1.951 & & & & & & \\
\hline \multirow{2}{*}{ Italy } & $22.01 .2020-11.03 .2020$ & -6.174 & -8.074 & -6.297 & \multirow{2}{*}{-0.720} & \multirow{2}{*}{-0.768} & \multirow{2}{*}{-0.753} & \multirow{2}{*}{0.518} & \multirow{2}{*}{0.589} & \multirow{2}{*}{0.567} \\
\hline & $12.03 .2020-14.04 .2020$ & -0.783 & -0.210 & -4.735 & & & & & & \\
\hline \multirow{2}{*}{ France } & $22.01 .2020-11.03 .2020$ & -2.665 & -5.698 & 0.353 & \multirow{2}{*}{-0.729} & \multirow{2}{*}{-0.759} & \multirow{2}{*}{-0.765} & \multirow{2}{*}{0.532} & \multirow{2}{*}{0.576} & \multirow{2}{*}{0.585} \\
\hline & $12.03 .2020-14.04 .2020$ & -1.581 & -1.692 & -1.387 & & & & & & \\
\hline \multirow{2}{*}{ Germany } & $22.01 .2020-11.03 .2020$ & -1.887 & -7.095 & 1.243 & \multirow{2}{*}{-0.597} & 0732 & 0730 & 0356 & 527 & 0538 \\
\hline & $12.03 .2020-14.04 .2020$ & -1.498 & -3.835 & -3.109 & & -0.733 & -0.734 & 0.356 & 0.537 & 0.538 \\
\hline uk & $22.01 .2020-11.03 .2020$ & -0.780 & -1.823 & -7.831 & & & & & & \\
\hline UK & $12.03 .2020-14.04 .2020$ & -3.561 & -3.487 & -2.391 & -0.638 & -0.751 & -0.725 & 0.407 & 0.564 & 0.525 \\
\hline Bolrium & $22.01 .2020-11.03 .2020$ & -2.623 & -4.972 & -9.560 & & & & & & 480 \\
\hline Betgium & $12.03 .2020-14.04 .2020$ & -0.532 & -2.966 & -2.499 & & & & & & 0.480 \\
\hline New Zealand & $28.02 .2020-14.04 .2020$ & -3.676 & -2.635 & -2.421 & -0.831 & -0.868 & -0.840 & 0.690 & 0.753 & 0.705 \\
\hline Turkey & $28.02 .2020-14.04 .2020$ & -6.312 & -6.547 & 0.000 & -0.617 & -0.680 & 0.000 & 0.380 & 0.462 & 0.000 \\
\hline
\end{tabular}

increased 2.76 times during the given period. As for the March 12 - April 14, the slot is already negative - 2.45 times. In the second phase of the spread of the virus (March 12 April 14), along with the $1 \%$ increase in the spread of the virus, the intensity of transit traffic decreased by 2.45 times. As for the correlation between the presented variables for the mentioned period, it was $-78.2 \%$, with its determination coefficient $\left(R^{\wedge} 2\right)$ is $61.12 \%$.

Table 1 shows the slope, correlation, and determination coefficients between pedestrians, traffic, transit intensity, and the prevalence of the virus by countries from January 22 to March 22 (March 11) and March 12 to April 14.

Table 1 shows that the slope and correlation coefficients are both positive and negative. From 22.01.2020 to 14.04.2020, the maximum value of the positive slope coefficient between the US pedestrian traffic ( 1.04 times) and the intensity of traffic (2,653 times) and the prevalence of the virus. And Spain has the highest positive ratio between the intensity of transit traffic and the spread of the virus in Spain (2,985 times). 22.01.2020 14.04.2020 The maximum value of the negative slope coefficient is between Turkey in terms of pedestrian traffic (-
6,312 times), Italy - in terms of traffic intensity (-8,074 times) and the prevalence of the virus. And the highest negative ratio between the transit traffic intensity and the prevalence of the virus in Belgium (-9.560 times).

During this period, the correlation coefficient in all presented directions is negative. New Zealand has the highest negative correlation between pedestrians $(-83.07 \%)$, vehicle traffic intensity $(86.8 \%)$ and the prevalence of the virus. Therefore, their determination coefficient $(R \wedge$ 2 ) was $69.01 \%$ and $75.34 \%$, respectively.

\section{CONCLUSION}

Social distancing and lock down will benefit the local and international community. Moreover, it has been suggested that evacuations may contribute to the international spread of a highly contagious disease of pandemic potential (9). Also to be considered, significant disparities in social determinants of health exist between the rural and urban areas. They are likely to increase mortality rate to COVID-19 if the disease reaches the 
rural areas. Deliberate and proactive measures are needed acutely to prevent the spread of this disease to rural areas. Contingency plans must be in place to deal with the disease should it eventually get there (10). "Contamination due to contact with the infected person" is the main responsible factor behind the pandemic COVID-19. Also, in this investigation we get an optimal model by which we can monitor the death from Coronavirus within the affected person continuously. By Doctors opinion, Literature review and Media Survey was selected three risk factors of Coronavirus namely Verbal contamination, contamination through eatables, and contamination due to contact with the infected person (11). The study showed that elderly patients and those with comorbidities are most susceptible for this infection, and will have the worst prognosis (12).

Thus in this study, in the conclusion it should be noted that the intensity of pedestrians, traffic and transit traffic during the study period, on average, after 15-20 days, affected the spread of the virus. If there was a positive slope and correlation coefficient between the variables presented in the period 22.01.2020 - 11.03.2020 (before the announcement of the pandemic), in the period 12.03.2020 - 14.04.2020 (after the announcement of the pandemic) the slope and correlation coefficients received negative values between the study variables, which indicates That on average, after 15-20 days, Due to the intensity of the movement, the center of the virus spreading is identified, and the intensity of the movement itself is decreased.

For future research, these correlation studies will play an important role in further influencing mobility trends in the prevention of various infectious diseases, as well as socioeconomic impact, health, and ecosystem sustainability in the post-epidemic period.

\section{REFERENCES}

1. Chen S, Yang J, Yang W, Wang C, Bärnighausen T. COVID-19 control in China during mass population movements at New Year. Lancet. 2020;395:764-6. https://doi.org/10.1016/ S0140-6736(20)30421-9

2. Gondauri D., Mikautadze E., Batiashvili M. Research on COVID-19 Virus Spreading Statistics based on the Examples of the Cases from Different Countries. Electron J Gen Med. 2020;17(4):em209. https://doi.org/10.29333/ejgm/7869
3. Kraemer M.U.G., Yang Ch., Gutierrez B., Wu Ch., Klein B., Pigott D.M., Open COVID-19 Data Working Group, Plessis L., Faria N.R., Li R., Hanage W.P., Brownstein J.S., Layan M., Vespignani A., Tian H., Dye Ch., Pybus O.G., Scarpino S.V. The effect of human mobility and control measures on the COVID-19 epidemic in China. Science. 2020; 25 Mar: eabb4218. https://doi.org/10.1126/science.abb4218 PMid: 32213647 PMCid:PMC7146642

4. Peeters Grietens K., Gryseels C., Dierickx S. et al. Characterizing Types of Human Mobility to Inform Differential and Targeted Malaria Elimination Strategies in Northeast Cambodia. Sci Rep. 2015; 5, 16837. https://doi.org/10.1038/srep16837 PMid:26593245 PMCid: PMC4655368

5. Massaro E., Kondor D. \& Ratti C. Assessing the interplay between human mobility and mosquito borne diseases in urban environments. Sci Rep. 2019; 9, 16911. https://doi. org/10.1038/s41598-019-53127-z PMid:31729435 PMCid: PMC6858332

6. Long NN, Khoi BH. An Empirical Study about the Intention to Hoard Food during COVID-19 Pandemic. EURASIA J MATH SCI T. 2020;16(7), em1857. https://doi.org/ 10.29333/ejmste/8207

7. Schober P., Boer Ch., Schwarte L.A. Correlation Coefficients: Appropriate Use and Interpretation. Anesthesia \& Analgesia. 2018; 126(5):1763-1768 https://doi. org/10.1213/ANE.0000000000002864 PMid:29481436

8. Mukaka MM. Statistics corner: A guide to appropriate use of correlation coefficient in medical research. Malawi Med J. 2012; 24(3):69-71. PMID: 23638278

9. Musinguzi G., Asamoah BO. The Science of Social Distancing and Total Lock Down: Does it Work? Whom does it Benefit?. Electron J Gen Med. 2020; 17(6):em230. https://doi.org/10.29333/ejgm/7895

10. Ameh, G. G., Njoku, A., Inungu, J. and Younis, M. (2020). Rural America and Coronavirus Epidemic: Challenges and Solutions. European Journal of Environment and Public Health, 4(2), em0040. https://doi.org/10.29333/ejeph/8200

11. Majumder P, Biswas $\mathrm{P}$, Majumder S. Application of New TOPSIS Approach to Identify the Most Significant Risk Factor and Continuous Monitoring of Death of COVID-19. Electron J Gen Med. 2020;17(6): em234. https://doi.org/ 10.29333/ejgm/7904

12. Dakhil ZA, Farhan HA. Cardiovascular Impacts of COVID-19 Pandemic: From Presentation to Management: Current and Future Perspectives. J Clin Exp Invest. 2020;11(3):em00739. https://doi.org/10.5799/jcei/7941 